Provided for non-commercial research and educational use only. Not for reproduction or distribution or commercial use.

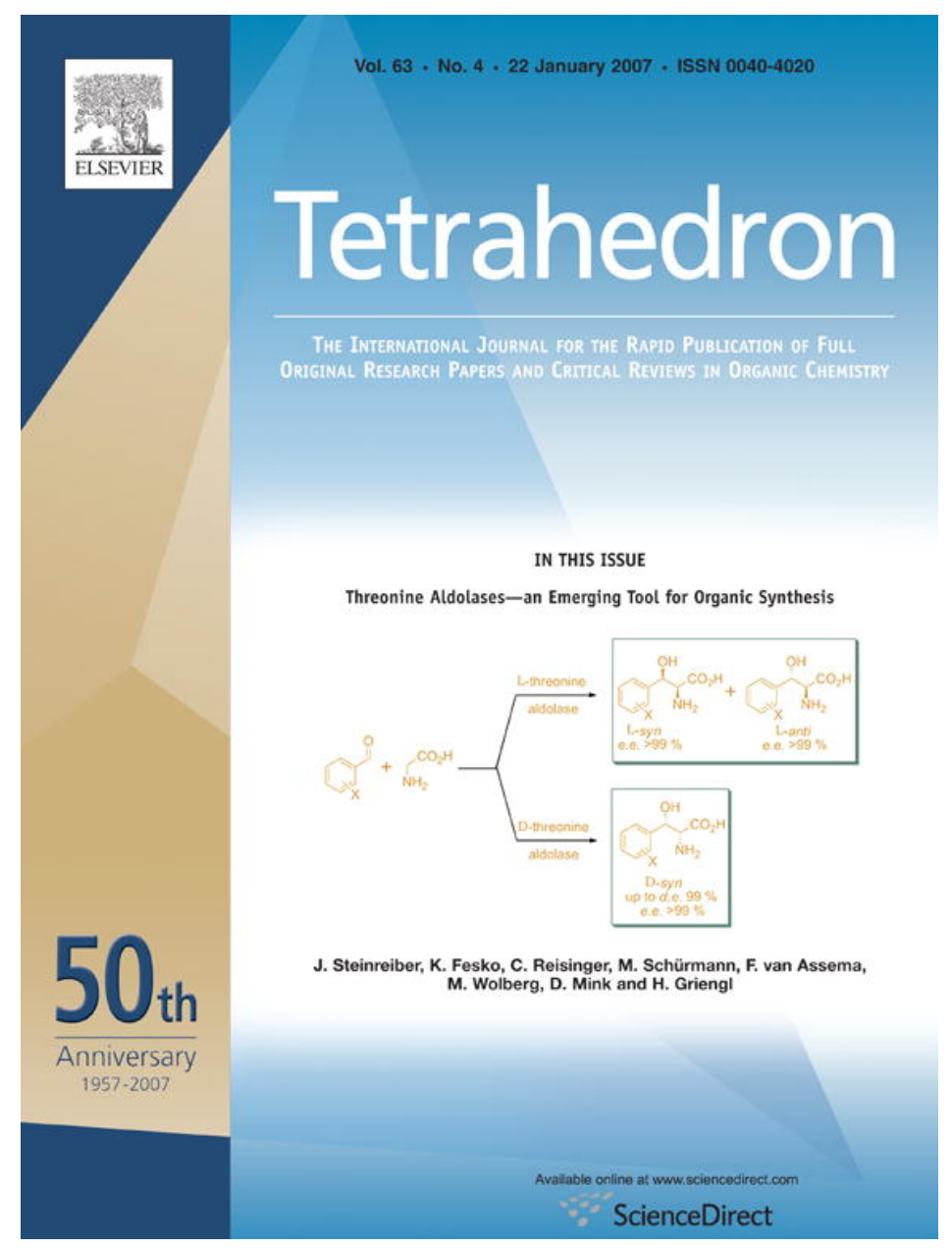

This article was originally published in a journal published by Elsevier, and the attached copy is provided by Elsevier for the author's benefit and for the benefit of the author's institution, for non-commercial research and educational use including without limitation use in instruction at your institution, sending it to specific colleagues that you know, and providing a copy to your institution's administrator.

All other uses, reproduction and distribution, including without limitation commercial reprints, selling or licensing copies or access,

or posting on open internet sites, your personal or institution's website or repository, are prohibited. For exceptions, permission may be sought for such use through Elsevier's permissions site at: 


\title{
Facile synthesis of azocino[4,3-b]indoles by ring-closing metathesis
}

\author{
M.-Lluïsa Bennasar, ${ }^{*}$ Ester Zulaica, Daniel Solé and Sandra Alonso \\ Laboratory of Organic Chemistry, Faculty of Pharmacy, University of Barcelona, Barcelona 08028, Spain \\ Received 24 October 2006; revised 13 November 2006; accepted 16 November 2006 \\ Available online 11 December 2006
}

\begin{abstract}
The azocino[4,3- $b$ ]indole system, tricyclic substructure of the indole alkaloids apparicine and ervaticine, is efficiently assembled by ring-closing metathesis of 2-allyl-3-(allylaminomethyl)indoles. The metathesis sites are introduced into the indole nucleus by reductive amination of a 3-formyl derivative with allylamine, followed by $\alpha$-lithiation with subsequent electrophilic trapping with acrolein. (c) 2006 Elsevier Ltd. All rights reserved.
\end{abstract}

\section{Introduction}

Ruthenium-catalyzed ring-closing metathesis $(\mathrm{RCM})^{1}$ has emerged as a powerful tool for the construction of a great variety of carbo- and heterocycles from acyclic precursors. ${ }^{2}$ In particular, the RCM methodology has turned out to be very useful for the synthesis of medium-sized rings, ${ }^{3}$ which is generally problematic due to disfavored entropic factors and transannular interactions. Our interest in the development of indole annulation methodologies led us to consider RCM reactions of indole-containing dienes ${ }^{4,5}$ for the efficient construction of medium-sized indolo 2,3-fused carboand azacycles, which are common structural arrangements in many natural and synthetic bioactive compounds. ${ }^{6}$ In this paper we report a direct synthetic approach to the azocino[4,3-b]indole system I by RCM of appropriate 2,3-dialkenylindoles incorporating a nitrogen atom in the tether linking the two double bonds (Scheme 1). It should be noted that I constitutes the tricyclic substructure of apparicine, ${ }^{7}$ an

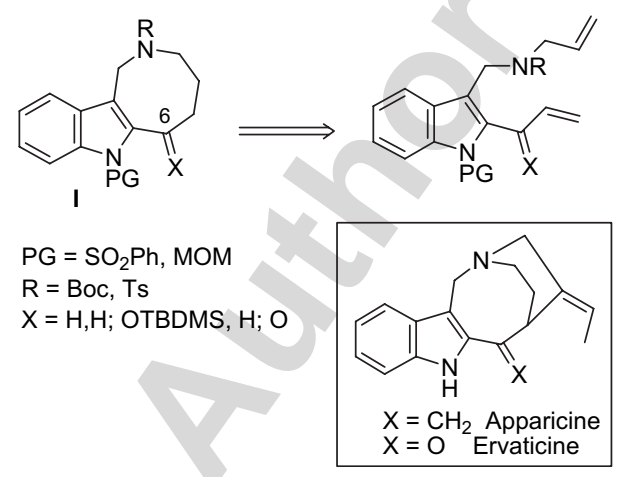

Scheme 1. Synthetic plan.

Keywords: Ring-closing metathesis; Indole; Indole alkaloids.

* Corresponding author. Tel.: +34934024540; fax: +34934024539; e-mail: bennasar@ub.edu indole alkaloid known for 40 years but still awaiting its first total synthesis, ${ }^{8}$ and also the unprecedented 2-acylindole analogue ervaticine. ${ }^{9}$

\section{Results and discussion}

We set out to explore the feasibility of the protocol using model RCM precursors unfunctionalized at the benzylic indole $\alpha$-position, such as 2-allyl-3-(allylaminomethyl)indoles $3 .{ }^{10}$ For the preparation of these substrates, a fast formylation-reductive amination sequence starting from the known 2-allylindole $\mathbf{1}^{11}$ was envisaged (Scheme 2 ). A strong electron-withdrawing benzenesulfonyl group was placed at the indole nitrogen to guarantee the stability of the proposed gramine-type intermediates. Thus, Friedel-Crafts reaction of 1 with $\mathrm{Cl}_{2} \mathrm{CHOMe}$ in the presence of $\mathrm{TiCl}_{4}$ gave the aldehyde $2(90 \%)$, which was subjected to reductive amination with allylamine, followed by reaction of the resulting secondary amine with $(t-\mathrm{BuOCO})_{2} \mathrm{O}$ or $\mathrm{TsCl}$. In this manner, the required RCM substrates $\mathbf{3 a}$ or $\mathbf{3 b}$, bearing different

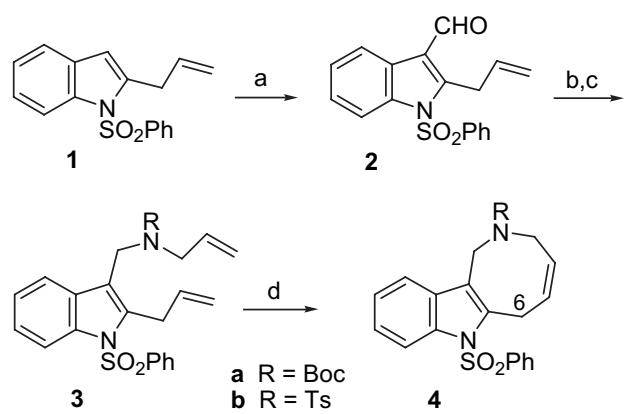

Scheme 2. Reagents and conditions: (a) $\mathrm{Cl}_{2} \mathrm{CHOMe}, \mathrm{TiCl}_{4}, \mathrm{CH}_{2} \mathrm{Cl}_{2}$, $-78{ }^{\circ} \mathrm{C}, 2 \mathrm{~h}, 90 \%$; (b) allylamine, $\mathrm{NaBH}(\mathrm{OAc})_{3}, \mathrm{AcOH}$, rt, overnight; (c) $(t-\mathrm{BuOCO})_{2} \mathrm{O}, 4: 1 \mathrm{MeOH}-\mathrm{Et}_{3} \mathrm{~N}$, reflux, $4 \mathrm{~h}, 65 \%(3 \mathrm{a})$ or $\mathrm{TsCl}, \mathrm{Et}_{3} \mathrm{~N}$, $\mathrm{CH}_{2} \mathrm{Cl}_{2}$, rt, overnight $65 \%(3 \mathbf{b})$; (d) $\left(\mathrm{PCy}_{3}\right)_{2}(\mathrm{Cl})_{2} \mathrm{Ru}=\mathrm{CHPh}, \mathrm{CH}_{2} \mathrm{Cl}_{2}$, reflux, overnight, $60 \%(4 \mathbf{a}), 89 \%(4 \mathbf{b})$. 
protecting groups at the aliphatic nitrogen, were obtained in $65 \%$ overall yield from 2 . Satisfactorily, ring closure of the $\mathrm{N}$-Boc diene 3a took place in refluxing dichloromethane in the presence of the first generation Grubbs catalyst to give the azocino[4,3- $b]$ indole $4 a$ in $60 \%$ yield. The $N$-tosyl derivative $\mathbf{3 b}$ proved to be a better substrate as it led to $\mathbf{4 b}$ in a higher yield (89\%).

With model azocino[4,3- $b$ ]indoles in hand, we sought to elaborate C-6 functionalized derivatives simply by extending the chemistry outlined above to an $O$-protected 2-(1hydroxyallyl)indole. To this end, we selected silyl ether $\mathbf{6}$, which was easily prepared from aldehyde $\mathbf{5},{ }^{12}$ by reaction with vinylmagnesium bromide followed by protection of the resulting alcohol with tert-butyldimethylsilyl chloride (63\% overall yield, Scheme 3). Disappointingly, we were not able to introduce the formyl group needed for the reductive amination step since $\mathbf{6}$ gave only a complex mixture upon subjection to the above Friedel-Crafts protocol.

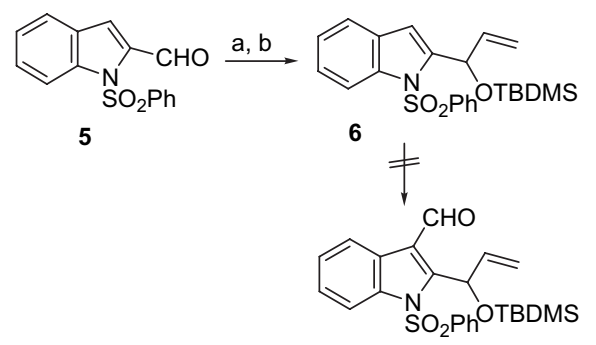

Scheme 3. Reagents and conditions: (a) $\mathrm{BrMgCH}=\mathrm{CH}_{2}$, THF, $-78^{\circ} \mathrm{C}-\mathrm{rt}$, overnight; (b) TBDMSCl, DMF, imidazole, $55^{\circ} \mathrm{C}$, overnight, $63 \%$.

This unsuccessful result prompted us to change the order of the synthetic steps. Functionalization at the 2-position of a properly 3 -substituted indole by $\alpha$-metalation followed by electrophilic trapping seemed to be the logical solution. With this aim, we focused our attention on 3-(aminomethyl)indoles $\mathbf{8}$ and $\mathbf{9}$, which were available from indole-3-carbaldehyde $7^{13}$ through reductive amination techniques, using tosylamine or, as above, allylamine followed by acylation (Scheme 4). Unfortunately, treatment of these substrates with either LDA, sec-BuLi or tert-BuLi in THF under a variety of experimental conditions, followed by addition of DMF, HCOOMe, or acrolein led to the recovery of the starting product.

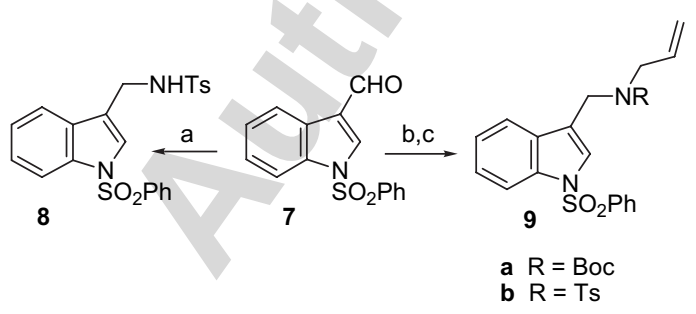

Scheme 4. Reagents and conditions: (a) $\mathrm{TsNH}_{2}$, toluene, reflux, $24 \mathrm{~h}$, then $\mathrm{NaBH}_{4}, \mathrm{MeOH}, \mathrm{rt}, 24 \mathrm{~h}, 70 \%$; (b) allylamine, $\mathrm{NaBH}(\mathrm{OAc})_{3}, \mathrm{AcOH}, \mathrm{rt}$, overnight; (c) $(t-\mathrm{BuOCO})_{2} \mathrm{O}, 4: 1 \mathrm{MeOH}-\mathrm{Et}_{3} \mathrm{~N}$, reflux, $4 \mathrm{~h}, 68 \%$ (9a) or TsCl, $\mathrm{Et}_{3} \mathrm{~N}, \mathrm{CH}_{2} \mathrm{Cl}_{2}$, rt, overnight $67 \%$ (9b).

We reasoned that the replacement of the indole protecting group by a methoxymethyl (MOM) group could facilitate the $\alpha$-lithiation, despite a probable reduction in stability of some synthetic intermediates due to the lower electronwithdrawing character of this moiety. Thus, we turned to aldehyde $\mathbf{1 0}^{14}$ (Scheme 5), which was converted into the allylaminomethyl derivatives $\mathbf{1 1}$ under the usual conditions. As the tosyl compound 11b partially decomposed under chromatographic purification, we decided to continue the synthesis only with the more stable $N$-Boc derivative 11a, which could be isolated in a reproducible $72 \%$ yield.

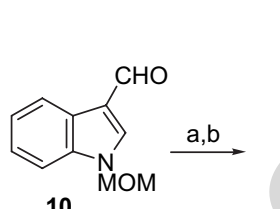

10

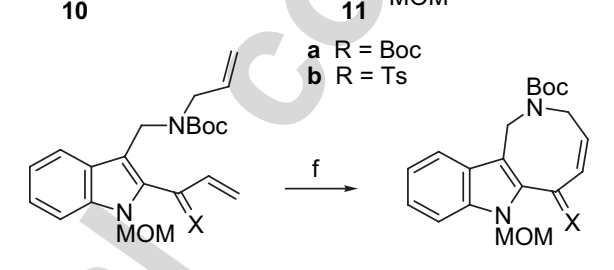
$13 X=\mathrm{H}$, OTBDMS $\longrightarrow 14 x=0$

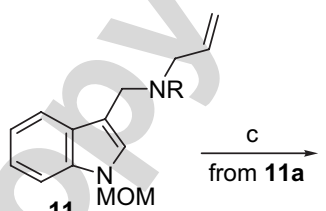
11 $15 X=H$, OTBDMS
$16 x=O$

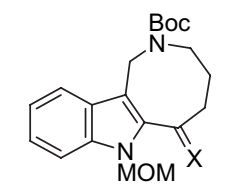

$17 \mathrm{X}=\mathrm{H}$, OTBDMS $18 X=0$
$12 x=\mathrm{H}, \mathrm{OH}$

Scheme 5. Reagents and conditions: (a) allylamine, $\mathrm{NaBH}(\mathrm{OAc})_{3}, \mathrm{AcOH}$, rt, overnight; (b) $(t \text {-BuOCO) })_{2} \mathrm{O}, 4: 1 \mathrm{MeOH}-\mathrm{Et}_{3} \mathrm{~N}$, reflux, $4 \mathrm{~h}, 72 \%$ (11a) or TsCl, $\mathrm{Et}_{3} \mathrm{~N}, \mathrm{CH}_{2} \mathrm{Cl}_{2}$, rt, overnight (11b); (c) $t$-BuLi, THF, $-78^{\circ} \mathrm{C}, 2 \mathrm{~h}$, then acrolein, $-78^{\circ} \mathrm{C}, 3.5 \mathrm{~h}, 79 \%$; (d) $\mathrm{MnO}_{2}, \mathrm{CH}_{2} \mathrm{Cl}_{2}, \mathrm{rt}, 60 \mathrm{~h}, 64 \%$; (e) TBDMSCl, DMF, imidazole, $55^{\circ} \mathrm{C}$, overnight, 64\%; (f) $\left(\mathrm{PCy}_{3}\right)_{2}(\mathrm{Cl})_{2} \mathrm{Ru}=$ $\mathrm{CHPh}, \mathrm{CH}_{2} \mathrm{Cl}_{2}$, reflux, overnight, $85 \%(\mathbf{1 5})$ or $(\mathrm{Im})(\mathrm{PCy})(\mathrm{Cl})_{2} \mathrm{Ru}=\mathrm{CHPh}$, $\mathrm{CH}_{2} \mathrm{Cl}_{2}$, rt, overnight, $86 \%$ (16); (g) $\mathrm{H}_{2}, \mathrm{Pd} / \mathrm{C}, \mathrm{MeOH}, 12 \mathrm{~h}, 80 \%$ (17), $82 \%$ (18).

We were pleased to find that the desired $\alpha$-lithiation did take place from 11a upon treatment with tert-BuLi in THF at $-78{ }^{\circ} \mathrm{C}$. After quenching with acrolein, the unstable alcohol 12 was isolated (79\%) and immediately protected as the tert-butyldimethylsilyl ether $\mathbf{1 3}(64 \%)$ or, alternatively, oxidized with $\mathrm{MnO}_{2}(64 \%)$ to the ketone 14. Satisfactorily, when 13 was subjected to the previously used RCM conditions (first generation Grubbs catalyst in refluxing dichloromethane) the expected tricyclic compound $\mathbf{1 5}$ was obtained in good yield (85\%). However, no cyclization was observed from ketone $\mathbf{1 4}$ under the above protocol, probably due to the presence of an electron-poor double bond, and only dimeric products coming from intermolecular metathesis reactions were formed. This problem was circumvented simply by using the more efficient second generation Grubbs catalyst at room temperature, leading to tricyclic ketone $\mathbf{1 6}$ in $86 \%$ yield. Finally, the saturated forms of the eight-membered heterocycles $\mathbf{1 7}$ and $\mathbf{1 8}$ were obtained by catalytic hydrogenation over $\mathrm{Pd} / \mathrm{C}$.

\section{Conclusion}

We have developed a new synthetic route to the azocino[4,3$b$ indole system ${ }^{15,16}$ relying on RCM of 2-allyl-3-(allylaminomethyl)indoles. The efficiency of the cyclization 
combined with the easy preparation of the dienic precursors from simple indolic derivatives make this strategy attractive for the construction of medium-sized indolo 2,3-fused carbo- and azacycles, which are scaffolds found in many bioactive compounds.

\section{Experimental}

\subsection{General methods}

All nonaqueous reactions were performed under an argon atmosphere. All solvents were dried by standard methods. Reaction courses and product mixtures were routinely monitored by TLC on silica gel (precoated $\mathrm{F}_{254}$ Merck plates). Drying of organic extracts was carried out over anhydrous $\mathrm{Na}_{2} \mathrm{SO}_{4}$. The solvents were evaporated under reduced pressure with a rotary evaporator. Flash chromatography was carried out on $\mathrm{SiO}_{2}$ (silica gel 60, SDS, 0.04-0.06 mm). Melting points are uncorrected. Unless otherwise indicated, NMR spectra were recorded in $\mathrm{CDCl}_{3}$ at $300 \mathrm{MHz}\left({ }^{1} \mathrm{H}\right)$ or $75.4 \mathrm{MHz}\left({ }^{13} \mathrm{C}\right)$ using $\mathrm{Me}_{4} \mathrm{Si}$ as an internal reference.

\subsubsection{2-Allyl-1-(phenylsulfonyl)-3-indolecarbaldehyde} (2). 2-Allylindole $\mathbf{1}^{11}(0.7 \mathrm{~g}, 2.3 \mathrm{mmol})$ in $\mathrm{CH}_{2} \mathrm{Cl}_{2}(6 \mathrm{~mL})$ was added to a solution of $\mathrm{TiCl}_{4}(0.51 \mathrm{~mL}, 4.7 \mathrm{mmol})$ and $\mathrm{Cl}_{2} \mathrm{CHOCH}_{3} \quad(0.4 \mathrm{~mL}, 4.7 \mathrm{mmol})$ in $\mathrm{CH}_{2} \mathrm{Cl}_{2} \quad(6 \mathrm{~mL})$ at $-78^{\circ} \mathrm{C}$, and the resulting mixture was stirred at $-78^{\circ} \mathrm{C}$ for $2 \mathrm{~h}$. The reaction mixture was diluted with $\mathrm{H}_{2} \mathrm{O}$, basified with $10 \%$ aqueous $\mathrm{Na}_{2} \mathrm{CO}_{3}$, and extracted with $\mathrm{CH}_{2} \mathrm{Cl}_{2}$. The organic extracts were dried and concentrated and the residue was purified by flash chromatography $(9: 1$ hexanes-AcOEt) to give aldehyde 2: $0.69 \mathrm{~g}(90 \%) ;{ }^{1} \mathrm{H}$ NMR $\delta 4.22(\mathrm{~m}, 2 \mathrm{H})$, $5.05(\mathrm{dm}, J=17.0,1.1 \mathrm{~Hz}, 1 \mathrm{H}), 5.12(\mathrm{dm}, J=10.0,1.1 \mathrm{~Hz}$, 1H), $6.05(\mathrm{~m}, 1 \mathrm{H}), 7.34-7.65(\mathrm{~m}, 5 \mathrm{H}), 7.86(\mathrm{~m}, 2 \mathrm{H}), 8.17$ $(\mathrm{m}, 1 \mathrm{H}), 8.22(\mathrm{~m}, 1 \mathrm{H}), 10.26(\mathrm{~s}, 1 \mathrm{H}) ;{ }^{13} \mathrm{C}$ NMR $\delta 29.5$ $\left(\mathrm{CH}_{2}\right), 114.3(\mathrm{CH}), 117.6\left(\mathrm{CH}_{2}\right), 119.5(\mathrm{C}), 121.4(\mathrm{CH})$, $125.1(\mathrm{CH}), 125.6(\mathrm{CH}), 126.0(\mathrm{C}), 126.6(2 \mathrm{CH}), 129.4$ $(2 \mathrm{CH}), 134.2(\mathrm{CH}), 134.4(\mathrm{CH}), 135.9(\mathrm{C}), 138.4(\mathrm{C}), 148.8$ (C), 185.6 (CO). Anal. Calcd for $\mathrm{C}_{18} \mathrm{H}_{15} \mathrm{NO}_{3} \mathrm{~S} \cdot 1 / 4 \mathrm{H}_{2} \mathrm{O}$ : C, $65.52 \% ; \mathrm{H}, 4.73 \%$; N, 4.24\%. Found: C, $65.81 \%$; $\mathrm{H}, 4.86 \%$; N, $4.12 \%$.

4.1.2. 2-Allyl-3-[( $N$-allyl- $N$-tert-butoxycarbonylamino)methyl]-1-(phenylsulfonyl)indole (3a). Allylamine $(0.15 \mathrm{~mL}$, $2.0 \mathrm{mmol}), \mathrm{NaBH}(\mathrm{OAc})_{3}(0.64 \mathrm{~g}, 3.0 \mathrm{mmol})$, and $\mathrm{AcOH}$ $(0.06 \mathrm{~mL}, 1.1 \mathrm{mmol})$ were successively added to aldehyde $2(0.33 \mathrm{~g}, 1.0 \mathrm{mmol})$ in $\mathrm{CH}_{2} \mathrm{Cl}_{2}(8 \mathrm{~mL})$, and the mixture was stirred at $\mathrm{rt}$ overnight. The reaction mixture was diluted with $\mathrm{H}_{2} \mathrm{O}$, basified with solid $\mathrm{Na}_{2} \mathrm{CO}_{3}$, and extracted with $\mathrm{CH}_{2} \mathrm{Cl}_{2}$. The organic extracts were dried and concentrated and the resulting residue was purified by flash chromatography (98:2 $\mathrm{CH}_{2} \mathrm{Cl}_{2}-\mathrm{MeOH}$ ) to give the secondary amine: $0.32 \mathrm{~g}$. This compound was dissolved in $\mathrm{MeOH}(8 \mathrm{~mL})$, treated with $\mathrm{Et}_{3} \mathrm{~N}(2 \mathrm{~mL})$ and $(t-\mathrm{BuOCO})_{2} \mathrm{O}(0.32 \mathrm{~g}, 1.46 \mathrm{mmol})$, and the resulting mixture was heated at reflux for $4 \mathrm{~h}$. The solvent was removed and the residue was dissolved in $\mathrm{CH}_{2} \mathrm{Cl}_{2}(15 \mathrm{~mL})$ and washed successively with $1 \mathrm{~N} \mathrm{HCl}$ and brine. The organic extracts were dried and concentrated and the residue was purified by flash chromatography $\left(\mathrm{CH}_{2} \mathrm{Cl}_{2}\right)$ to give $3 \mathrm{a}: 0.30 \mathrm{~g}(65 \%)$; ${ }^{1} \mathrm{H}$ NMR (5:1 mixture of rotamers, major rotamer) $\delta 1.49$ (s, 9H), 3.40 (br s, 2H), 3.83 (br d, $J=6.0 \mathrm{~Hz}, 2 \mathrm{H}), 4.55$ (s, 2H), $4.90(\mathrm{~m}, 2 \mathrm{H}), 5.01(\mathrm{~m}, 2 \mathrm{H}), 5.60(\mathrm{~m}, 1 \mathrm{H}), 5.95(\mathrm{~m}, 1 \mathrm{H})$, $7.20-7.65(\mathrm{~m}, 6 \mathrm{H}), 7.70(\mathrm{~m}, 2 \mathrm{H}), 8.20(\mathrm{~d}, J=7.5 \mathrm{~Hz}, 1 \mathrm{H})$; ${ }^{13} \mathrm{C}$ NMR (major rotamer) $\delta 28.4\left(3 \mathrm{CH}_{3}\right), 29.9\left(\mathrm{CH}_{2}\right), 38.5$ $\left(\mathrm{CH}_{2}\right), 47.0\left(\mathrm{CH}_{2}\right), 79.9(\mathrm{C}), 115.0(\mathrm{CH}), 115.9\left(\mathrm{CH}_{2}\right), 116.2$ $\left(\mathrm{CH}_{2}\right), 119.3(\mathrm{CH}), 123.7(\mathrm{CH}), 124.5(\mathrm{CH}), 126.2(2 \mathrm{CH})$, $129.0(2 \mathrm{CH}), 129.8(\mathrm{C}), 133.4(\mathrm{CH}), 133.5(\mathrm{CH}), 134.9$ (CH), 136.5 (C), 136.9 (C), 138.7 (C), 155.5 (CO); HRMS calcd for $\mathrm{C}_{26} \mathrm{H}_{30} \mathrm{~N}_{2} \mathrm{O}_{4} \mathrm{~S} 466.1926$, found 466.1932 .

4.1.3. 2-Allyl-3-[( $N$-allyl- $N$-tosylamino)methyl $]-1$-(phenylsulfonyl)indole (3b). Aldehyde $2(0.33 \mathrm{~g}, 1.0 \mathrm{mmol})$ was allowed to react as above with allylamine and the resulting secondary amine $(0.32 \mathrm{~g})$ was dissolved in $\mathrm{CH}_{2} \mathrm{Cl}_{2}(10 \mathrm{~mL})$ and treated with tosyl chloride $(0.19 \mathrm{~g}, 1.0 \mathrm{mmol})$ and $\mathrm{Et}_{3} \mathrm{~N}$ $(0.14 \mathrm{~mL}, 1.0 \mathrm{mmol})$ at $\mathrm{rt}$ overnight. The reaction mixture was diluted with $\mathrm{CH}_{2} \mathrm{Cl}_{2}$ and washed with $1 \mathrm{~N} \mathrm{HCl}$ and brine. The organic extracts were dried and concentrated and the residue was chromatographed (flash, $\mathrm{CH}_{2} \mathrm{Cl}_{2}$ ) to give $\mathbf{3 b}$ : $0.34 \mathrm{~g}(65 \%) ; \mathrm{mp} 118^{\circ} \mathrm{C}\left(\mathrm{Et}_{2} \mathrm{O}\right) ;{ }^{1} \mathrm{H}$ NMR $\delta 2.44(\mathrm{~s}, 3 \mathrm{H})$, $3.52(\mathrm{~d}, J=6.3 \mathrm{~Hz}, 2 \mathrm{H}), 3.76(\mathrm{dm}, J=6.0 \mathrm{~Hz}, 2 \mathrm{H}), 4.36(\mathrm{~s}$, $2 \mathrm{H}), 4.66(\mathrm{dd}, J=17.0,1.5 \mathrm{~Hz}, 1 \mathrm{H}), 4.72(\mathrm{dd}, J=10.0$, $1.5 \mathrm{~Hz}, 1 \mathrm{H}), 4.92(\mathrm{dd}, J=17.0,1.5 \mathrm{~Hz}, 1 \mathrm{H}), 4.99(\mathrm{dd}$, $J=10.0,1.5 \mathrm{~Hz}, 1 \mathrm{H}), 5.23(\mathrm{~m}, 1 \mathrm{H}), 5.93(\mathrm{~m}, 1 \mathrm{H}), 7.25-7.75$ $(\mathrm{m}, 12 \mathrm{H}), 8.20(\mathrm{~d}, J=7.5 \mathrm{~Hz}, 1 \mathrm{H}) ;{ }^{13} \mathrm{C} \mathrm{NMR} \delta 21.5\left(\mathrm{CH}_{3}\right)$, $29.9\left(\mathrm{CH}_{2}\right), 41.5\left(\mathrm{CH}_{2}\right), 49.4\left(\mathrm{CH}_{2}\right), 114.9(\mathrm{CH}), 115.5(\mathrm{C})$, $116.3\left(\mathrm{CH}_{2}\right), 118.1\left(\mathrm{CH}_{2}\right), 119.5(\mathrm{CH}), 123.8(\mathrm{CH}), 124.7$ $(\mathrm{CH}), 126.2(2 \mathrm{CH}), 127.2(2 \mathrm{CH}), 129.0(2 \mathrm{CH}), 129.1(\mathrm{C})$, $129.7(2 \mathrm{CH}), 132.4(\mathrm{CH}), 133.6(\mathrm{CH}), 134.7(\mathrm{CH}), 136.5$ (C), 137.2 (C), 139.2 (C), 139.7 (C), 143.4 (C). Anal. Calcd for $\mathrm{C}_{28} \mathrm{H}_{28} \mathrm{~N}_{2} \mathrm{O}_{4} \mathrm{~S}_{2}$ : C, 64.59\%; H, 5.41\%; N, 5.38\%. Found: C, 64.67\%; H, 5.43\%; N, 5.35\%.

4.1.4. 2-(tert-Butoxycarbonyl)-7-(phenylsulfonyl)-1,2,3,6tetrahydroazocino $[4,3-b]$ indole $(4 a) . \quad\left(\mathrm{PCy}_{3}\right)_{2}(\mathrm{Cl})_{2} \mathrm{Ru}=$ $\mathrm{CHPh}$ (first generation Grubbs catalyst, $10 \mathrm{~mol} \%$ ) was added under Ar to a solution of amine 3a $(90 \mathrm{mg}, 0.19 \mathrm{mmol})$ in anhydrous $\mathrm{CH}_{2} \mathrm{Cl}_{2}(5 \mathrm{~mL})$ and the resulting mixture was heated at reflux overnight. The reaction mixture was filtered and concentrated. Flash chromatography of the crude residue (1:1 hexanes-AcOEt) gave 4a: $50 \mathrm{mg}(60 \%) ;{ }^{1} \mathrm{H}$ NMR $(1: 1$ mixture of rotamers) $\delta 1.25$ and $1.44(2 \mathrm{~s}, 9 \mathrm{H}), 3.82(\mathrm{~m}, 2 \mathrm{H})$, 3.88 and $4.02(2 \mathrm{br} \mathrm{s}, 2 \mathrm{H}), 4.55$ and $4.68(2 \mathrm{~s}, 2 \mathrm{H}), 5.60$ and $5.71(2 \mathrm{~m}, 1 \mathrm{H}), 5.85(\mathrm{~m}, 1 \mathrm{H}), 7.22-7.53(\mathrm{~m}, 7 \mathrm{H}), 7.73(\mathrm{~m}$, $1 \mathrm{H}), 8.20(\mathrm{~m}, 1 \mathrm{H}) ;{ }^{13} \mathrm{C}$ NMR $\delta 23.4\left(\mathrm{CH}_{2}\right), 28.3\left(3 \mathrm{CH}_{3}\right)$, $42.6\left(\mathrm{CH}_{2}\right), 45.9$ and $46.5\left(\mathrm{CH}_{2}\right), 79.9(\mathrm{C}), 114.8(\mathrm{CH})$, $117.8(\mathrm{CH}), 118.6(\mathrm{C}), 123.3(\mathrm{CH}), 124.2(\mathrm{CH}), 126.2$ (2CH), $127.1(\mathrm{CH}), 128.2(\mathrm{CH}), 129.0(\mathrm{C}), 129.1(2 \mathrm{CH})$, $133.5(\mathrm{CH}), 136.0(2 \mathrm{C}), 139.0(\mathrm{C}), 155.0(\mathrm{CO})$. Anal. Calcd for $\mathrm{C}_{24} \mathrm{H}_{26} \mathrm{~N}_{2} \mathrm{O}_{4} \mathrm{~S} \cdot 1 / 2 \mathrm{H}_{2} \mathrm{O}$ : C, 64.36\%; H, 6.08\%; N, $6.25 \%$. Found: C, 64.36\%; H, 5.94\%; N, 6.12\%.

4.1.5. 7-(Phenylsulfonyl)-2-tosyl-1,2,3,6-tetrahydroazocino $[4,3-b]$ indole (4b). Operating as above, from amine 3b $(0.1 \mathrm{~g}, 0.19 \mathrm{mmol})$ 4b was obtained: $80 \mathrm{mg}(89 \%)$; ${ }^{1} \mathrm{H}$ NMR $\left({ }^{1} \mathrm{H}\right.$ COSY) $\delta 2.40$ (s, 3H, Me), $3.76(\mathrm{~d}$, $J=6.6 \mathrm{~Hz}, 2 \mathrm{H}, 3-\mathrm{H}), 3.98(\mathrm{~d}, J=6.9 \mathrm{~Hz}, 2 \mathrm{H}, 6-\mathrm{H}), 4.51$ (s, $2 \mathrm{H}, 1-\mathrm{H}), 5.42(\mathrm{~m}, 1 \mathrm{H}, 4-\mathrm{H}), 5.92(\mathrm{~m}, 1 \mathrm{H}, 5-\mathrm{H}), 7.20-7.70$ $(\mathrm{m}, 12 \mathrm{H}, \mathrm{Ar}), 8.20(\mathrm{~d}, J=7.5 \mathrm{~Hz}, 1 \mathrm{H}, 8-\mathrm{H}) ;{ }^{13} \mathrm{C} \mathrm{NMR}$ (Hetcor) $\delta 21.5\left(\mathrm{CH}_{3}\right), 25.0(\mathrm{C}-6), 42.1(\mathrm{C}-1), 45.0(\mathrm{C}-3)$, 114.8 (C-8), 115.3 (C-11b), 117.9 (C-11), 123.6 (C-10), 124.6 (C-9), 125.2 (C-4), $126.1(2 \mathrm{CH}), 127.0(2 \mathrm{CH}), 129.0$ (C-11a), $129.2(2 \mathrm{CH}), 129.6(2 \mathrm{CH}), 129.8$ (C-5), 133.7 (CH), 136.0 (2C), 136.4 (C), 138.8 (C), 143.3 (C); HRMS calcd for $\mathrm{C}_{26} \mathrm{H}_{24} \mathrm{~N}_{2} \mathrm{O}_{4} \mathrm{~S}_{2} 492.6118$, found 492.6110 . 
4.1.6. 2-[1-(tert-Butyldimethylsilyloxy)-2-propenyl]-1(phenylsulfonyl)indole (6). $\mathrm{BrMgCH}=\mathrm{CH}_{2}$ (1 M solution in THF, $2.96 \mathrm{mmol}$ ) was added to a solution of aldehyde $5^{12}(0.65 \mathrm{~g}, 2.28 \mathrm{mmol})$ in $\mathrm{THF}(15 \mathrm{~mL})$ at $-78^{\circ} \mathrm{C}$ and the resulting mixture was stirred at $\mathrm{rt}$ overnight. The reaction mixture was diluted with $10 \%$ aqueous $\mathrm{NH}_{4} \mathrm{Cl}$ and extracted with $\mathrm{Et}_{2} \mathrm{O}$. The organic extracts were dried and concentrated and the residue was purified by flash chromatography (9:1 hexanes-AcOEt) to give 1-(phenylsulfonyl)-2-(1-hydroxy2-propenyl)indole $(0.57 \mathrm{~g})$. This compound was dissolved in DMF $(5 \mathrm{~mL})$ and treated with TBDMSCl $(0.40 \mathrm{~g}$, $2.7 \mathrm{mmol})$ and imidazole $(0.30 \mathrm{~g}, 4.5 \mathrm{mmol})$ at $55^{\circ} \mathrm{C}$ overnight. The reaction mixture was diluted with $\mathrm{H}_{2} \mathrm{O}$ and extracted with $\mathrm{Et}_{2} \mathrm{O}$. The organic extracts were dried and concentrated. Flash chromatography (95:5 hexanes-AcOEt) of the residue gave 6: $0.61 \mathrm{~g}(63 \%) ;{ }^{1} \mathrm{H}$ NMR $\delta-0.01$ and $0.10(2 \mathrm{~s}, 6 \mathrm{H}), 0.95(\mathrm{~s}, 9 \mathrm{H}), 5.15(\mathrm{~d}, J=10.0 \mathrm{~Hz}, 1 \mathrm{H}), 5.40$ $(\mathrm{d}, J=15 \mathrm{~Hz}, 1 \mathrm{H}), 5.90($ br s, $1 \mathrm{H}), 6.20(\mathrm{~m}, 1 \mathrm{H}), 6.85(\mathrm{~s}$, $1 \mathrm{H}), 7.25-7.50(\mathrm{~m}, 6 \mathrm{H}), 7.60(\mathrm{~m}, 2 \mathrm{H}), 8.15(\mathrm{~d}, J=7.5 \mathrm{~Hz}$, $1 \mathrm{H}) ;{ }^{13} \mathrm{C}$ NMR $\delta-4.93$ and $-4.78\left(2 \mathrm{CH}_{3}\right), 18.3(\mathrm{C}), 25.8$ $\left(3 \mathrm{CH}_{3}\right), 69.5(\mathrm{CH}), 109.8(\mathrm{CH}), 114.5\left(\mathrm{CH}_{2}\right), 115.0(\mathrm{CH})$, $120.8(\mathrm{CH}), 123.8(\mathrm{CH}), 124.3(\mathrm{CH}), 126.3(2 \mathrm{CH}), 129.0$ (2CH), $129.9(\mathrm{C}), 133.6(\mathrm{CH}), 137.7(\mathrm{C}), 138.6(\mathrm{C}), 139.8$ $(\mathrm{CH}), \quad 144.4$ (C); HRMS calcd for $\mathrm{C}_{23} \mathrm{H}_{29} \mathrm{NO}_{3} \mathrm{SSi}$ 427.1637, found 427.1640 .

4.1.7. 1-(Phenylsulfonyl)-3-(tosylaminomethyl)indole (8). A solution of aldehyde $7^{13}(0.5 \mathrm{~g}, 1.75 \mathrm{mmol})$ and tosylamine $(0.9 \mathrm{~g}, 5.25 \mathrm{mmol})$ in dry toluene $(15 \mathrm{~mL})$ was heated at reflux (Dean-Stark) for $24 \mathrm{~h}$. The solvent was removed and the residue was dissolved in $\mathrm{MeOH}(10 \mathrm{~mL})$ and treated with $\mathrm{NaBH}_{4}(66 \mathrm{mg}, 1.75 \mathrm{mmol})$ at $\mathrm{rt}$ for $24 \mathrm{~h}$. The solvent was removed and the residue was diluted with $\mathrm{H}_{2} \mathrm{O}$ and extracted with $\mathrm{Et}_{2} \mathrm{O}$. The organic extracts were dried and concentrated and the resulting residue was purified by flash chromatography $(6: 4$ hexanes-AcOEt) to give tosylamine $\mathbf{8}$ : $0.54 \mathrm{~g}(70 \%)$; ${ }^{1} \mathrm{H} \mathrm{NMR}\left(\mathrm{CDCl}_{3}, 300 \mathrm{MHz}\right) \delta 2.41(\mathrm{~s}, 3 \mathrm{H})$, $4.22(\mathrm{~d}, J=6.0 \mathrm{~Hz}, 1 \mathrm{H}), 5.04($ br s, 2H), 7.20-7.90 (m, $13 \mathrm{H}) ;{ }^{13} \mathrm{C}$ NMR $\delta 21.5\left(\mathrm{CH}_{3}\right), 38.6\left(\mathrm{CH}_{2}\right), 113.4(\mathrm{CH})$, $117.6(\mathrm{C}), 119.6(\mathrm{CH}), 123.3(\mathrm{CH}), 124.5(\mathrm{CH}), 125.0$ $(\mathrm{CH}), 126.3(2 \mathrm{CH}), 126.6(2 \mathrm{CH}), 129.2(2 \mathrm{CH}), 129.6$ (2CH, C), $133.8(\mathrm{CH}), 136.1(\mathrm{C}), 137.8(\mathrm{C}), 138.9$ (C), 143.4 (C); HRMS calcd for $\mathrm{C}_{22} \mathrm{H}_{20} \mathrm{~N}_{2} \mathrm{O}_{4} \mathrm{~S}_{2} 440.0864$, found 440.0857 .

4.1.8. 3-[(N-Allyl- $N$-tert-butoxycarbonyl)aminomethyl]1-(phenylsulfonyl)indole (9a). Allylamine $(0.34 \mathrm{~mL}$, $4.6 \mathrm{mmol}), \mathrm{NaBH}(\mathrm{OAc})_{3}(1.46 \mathrm{~g}, 6.9 \mathrm{mmol})$, and $\mathrm{AcOH}$ $(0.13 \mathrm{~mL}, 2.3 \mathrm{mmol})$ were successively added to aldehyde $7(0.65 \mathrm{~g}, 2.3 \mathrm{mmol})$ in $\mathrm{CH}_{2} \mathrm{Cl}_{2}(15 \mathrm{~mL})$ and the resulting mixture was stirred at $\mathrm{rt}$ overnight. The reaction mixture was diluted with $\mathrm{H}_{2} \mathrm{O}$, basified with $10 \%$ aqueous $\mathrm{Na}_{2} \mathrm{CO}_{3}$, and extracted with $\mathrm{CH}_{2} \mathrm{Cl}_{2}$. The organic extracts were dried and concentrated. Flash chromatography of the residue (98:2 $\left.\mathrm{CH}_{2} \mathrm{Cl}_{2}-\mathrm{MeOH}\right)$ gave the secondary amine $(0.5 \mathrm{~g})$. This compound was dissolved in $\mathrm{MeOH}(16 \mathrm{~mL})$ and treated with $(t-\mathrm{BuOCO})_{2} \mathrm{O}(0.57 \mathrm{~g}, 2.65 \mathrm{mmol})$ and $\mathrm{Et}_{3} \mathrm{~N}(7.4 \mathrm{~mL}$, $5.3 \mathrm{mmol})$. After the mixture was heated at reflux for $4 \mathrm{~h}$, the solvent was removed and the residue was diluted with $\mathrm{CH}_{2} \mathrm{Cl}_{2}$ and washed with $1 \mathrm{~N} \mathrm{HCl}$ and brine. The organic extracts were dried and concentrated and the resulting residue was chromatographed (flash, 95:5 hexanes-AcOEt) to give 9a: $0.66 \mathrm{~g}(68 \%) ;{ }^{1} \mathrm{H}$ NMR $\delta 1.48$ (s, 9H), 3.70 (br s,
2H), 4.52 (br s, 2H), $5.10(\mathrm{~m}, 2 \mathrm{H}), 5.65(\mathrm{~m}, 1 \mathrm{H}), 7.20$ $7.65(\mathrm{~m}, 7 \mathrm{H}), 7.85(\mathrm{~m}, 2 \mathrm{H}), 8.05(\mathrm{~d}, J=7.5 \mathrm{~Hz}, 1 \mathrm{H})$; ${ }^{13} \mathrm{C}$ NMR $\delta 28.3\left(3 \mathrm{CH}_{3}\right), 40.8\left(\mathrm{CH}_{2}\right), 48.0\left(\mathrm{CH}_{2}\right), 79.9$ (C), $113.5(\mathrm{CH}), 116.3\left(\mathrm{CH}_{2}\right), 119.6(\mathrm{CH}), 120.3(\mathrm{CH})$, $123.2(\mathrm{CH}), 124.6(\mathrm{CH}), 124.8(\mathrm{CH}), 126.5(2 \mathrm{CH}), 128.8$ (C), $129.1(2 \mathrm{CH}), 133.4(\mathrm{CH}), 133.7(\mathrm{CH}), 135.3(\mathrm{C})$, 137.9 (C), 155.3 (CO); HRMS calcd for $\mathrm{C}_{23} \mathrm{H}_{26} \mathrm{~N}_{2} \mathrm{O}_{4} \mathrm{~S}$ 426.1613, found 426.1610 .

4.1.9. 3-[( $N$-Allyl- $N$-tosyl)aminomethyl]-1-(phenylsulfonyl)indole (9b). Aldehyde $7(0.65 \mathrm{~g}, 2.3 \mathrm{mmol})$ was allowed to react as above with allylamine and the resulting secondary amine was dissolved in $\mathrm{CH}_{2} \mathrm{Cl}_{2}(12 \mathrm{~mL})$ and treated with tosyl chloride $(0.33 \mathrm{~g}, 1.75 \mathrm{mmol})$ and $\mathrm{Et}_{3} \mathrm{~N}$ $(0.25 \mathrm{~mL}, 1.75 \mathrm{mmol})$ at $\mathrm{rt}$ overnight. The reaction mixture was diluted with $\mathrm{CH}_{2} \mathrm{Cl}_{2}$ and washed with $1 \mathrm{~N} \mathrm{HCl}$ and brine prior to drying and solvent evaporation. The resulting residue was purified by flash chromatography $(1: 1$ hexanes$\left.\mathrm{CH}_{2} \mathrm{Cl}_{2}\right)$ to give 9b: $0.74 \mathrm{~g}(67 \%) ; \mathrm{mp} 108{ }^{\circ} \mathrm{C}\left(\mathrm{Et}_{2} \mathrm{O}\right)$; ${ }^{1} \mathrm{H}$ NMR $\delta 2.44(\mathrm{~s}, 3 \mathrm{H}), 3.70(\mathrm{~d}, J=6.3 \mathrm{~Hz}, 2 \mathrm{H}), 4.43(\mathrm{~s}$, 2H), $4.90(\mathrm{~m}, 2 \mathrm{H}), 5.37(\mathrm{~m}, 1 \mathrm{H}), 7.25-7.95(\mathrm{~m}, 13 \mathrm{H}), 8.01$ $(\mathrm{d}, J=7.5 \mathrm{~Hz}, 1 \mathrm{H}) ;{ }^{13} \mathrm{C}$ NMR $\delta 21.4\left(\mathrm{CH}_{3}\right), 41.8\left(\mathrm{CH}_{2}\right)$, $49.5\left(\mathrm{CH}_{2}\right), 113.3(\mathrm{CH}), 117.2(\mathrm{C}), 118.9\left(\mathrm{CH}_{2}\right), 120.1$ $(\mathrm{CH}), 123.4(\mathrm{CH}), 125.0(\mathrm{CH}), 125.3(\mathrm{CH}), 126.4(2 \mathrm{CH})$, $126.9(2 \mathrm{CH}), 129.1(2 \mathrm{CH}), 129.5(\mathrm{C}), 129.6(2 \mathrm{CH}), 131.9$ (CH), 133.7 (CH), 135.1 (C), 136.7 (C), 137.7 (C), 143.4 (C). Anal. Calcd for $\mathrm{C}_{25} \mathrm{H}_{24} \mathrm{~N}_{2} \mathrm{O}_{4} \mathrm{~S}_{2}$ : C, $62.48 \% ; \mathrm{H}, 5.03 \%$; N, $5.82 \%$. Found: C, $62.58 \%$; H, 5.21\%; N, 5.69\%.

4.1.10. 3-[( $N$-Allyl- $N$-tert-butoxycarbonyl)aminomethyl]1-(methoxymethyl)indole (11a). Aldehyde $\mathbf{1 0}^{14}(1.75 \mathrm{~g}$, $9.2 \mathrm{mmol})$ in $\mathrm{CH}_{2} \mathrm{Cl}_{2}(30 \mathrm{~mL})$ was allowed to react with allylamine $(1.41 \mathrm{~mL}, 18.8 \mathrm{mmol})$ and then with $(t-\mathrm{BuOCO})_{2} \mathrm{O}$ $(3.67 \mathrm{~g}, 16.8 \mathrm{mmol})$ as described in Section 4.1.8. After work-up and flash chromatography (7:3 hexanes-AcOEt), 11a was obtained: $2.20 \mathrm{~g}(72 \%) ;{ }^{1} \mathrm{H}$ NMR $\left(50{ }^{\circ} \mathrm{C}\right) \delta 1.50$ (s, 9H), 3.21 (s, 3H), 3.74 (br s, 2H), 4.59 (s, 2H), 5.10 (m, $2 \mathrm{H}), 5.47(\mathrm{~s}, 2 \mathrm{H}), 5.73(\mathrm{~m}, 1 \mathrm{H}), 7.08(\mathrm{~s}, 1 \mathrm{H}), 7.10-7.30$ (m, 2H), $7.46(\mathrm{~d}, J=8.0 \mathrm{~Hz}, 1 \mathrm{H}), 7.69(\mathrm{~d}, J=7.5 \mathrm{~Hz}, 1 \mathrm{H})$; ${ }^{13} \mathrm{C}$ NMR $\left(50{ }^{\circ} \mathrm{C}\right) \delta 28.5\left(3 \mathrm{CH}_{3}\right), 40.6\left(\mathrm{CH}_{2}\right), 47.9\left(\mathrm{CH}_{2}\right)$, $55.8\left(\mathrm{CH}_{3}\right), 77.3\left(\mathrm{CH}_{2}\right), 79.6(\mathrm{C}), 109.8(\mathrm{CH}), 113.0(\mathrm{C})$, $116.0\left(\mathrm{CH}_{2}\right), 119.6(\mathrm{CH}), 120.1(\mathrm{CH}), 122.4(\mathrm{CH}), 127.1$ (CH), $128.2(\mathrm{C}), 134.1(\mathrm{CH}), 136.9(\mathrm{C}), 155.4(\mathrm{CO})$. Anal. Calcd for $\mathrm{C}_{19} \mathrm{H}_{26} \mathrm{~N}_{2} \mathrm{O}_{3} \cdot 1 / 4 \mathrm{H}_{2} \mathrm{O}: \mathrm{C}, 68.14 \% ; \mathrm{H}, 7.97 \%$; N, $8.36 \%$. Found: C, $68.30 \%$; H, 8.07\%; N, 8.26\%.

4.1.11. 3-[( $N$-Allyl- $N$-tert-butoxycarbonyl)aminomethyl]2-(1-hydroxy-2-propenyl)-1-(methoxymethyl)indole (12). tert-BuLi (1.7 M in pentane, $0.87 \mathrm{mmol})$ was slowly added under Ar to a solution of indole 11a $(0.22 \mathrm{~g}, 0.72 \mathrm{mmol})$ in anhydrous THF $(10 \mathrm{~mL})$ at $-78^{\circ} \mathrm{C}$, and the resulting solution was stirred for $2 \mathrm{~h}$ at $-78^{\circ} \mathrm{C}$. Then, acrolein $(0.11 \mathrm{~mL}, 1.8 \mathrm{mmol})$ was added and the mixture was stirred at $-78^{\circ} \mathrm{C}$ for $3.5 \mathrm{~h}$. The reaction mixture was poured into $10 \%$ aqueous $\mathrm{NH}_{4} \mathrm{Cl}$ and extracted with $\mathrm{Et}_{2} \mathrm{O}$. The organic extracts were dried and concentrated and the residue was purified by flash chromatography (8:2 hexanes-AcOEt) to give alcohol 12: $0.22 \mathrm{~g}$ (79\%, unstable oil); ${ }^{1} \mathrm{H}$ NMR (gHSQC,) $\delta 1.49\left(\mathrm{~s}, 9 \mathrm{H}, 3 \mathrm{CH}_{3}\right), 3.26(\mathrm{~s}, 3 \mathrm{H}, \mathrm{OMe}), 3.75$ $\left(\mathrm{m}, 2 \mathrm{H}, \mathrm{NCH}_{2} \mathrm{CH}=\right), 4.68$ and $4.73(2 \mathrm{~d}, J=15.3 \mathrm{~Hz}, 2 \mathrm{H}$, ind $\left.\mathrm{CH}_{2} \mathrm{~N}\right), 5.09\left(\mathrm{~m}, 1 \mathrm{H}, \mathrm{CH}_{2}=\right), 5.13\left(\mathrm{~m}, 1 \mathrm{H}, \mathrm{CH}_{2}=\right)$, $5.24\left(\mathrm{dm}, J=10.8 \mathrm{~Hz}, 1 \mathrm{H}, \mathrm{CH}_{2}=\right), 5.35(\mathrm{dm}, J=17.0 \mathrm{~Hz}$, $\left.1 \mathrm{H}, \mathrm{CH}_{2}=\right), 5.47$ and $5.62\left(2 \mathrm{~d}, J=10.8 \mathrm{~Hz}, 2 \mathrm{H}, \mathrm{CH}_{2} \mathrm{OMe}\right)$, 
$5.72(\mathrm{~m}, 2 \mathrm{H}, \mathrm{CHOH}, \mathrm{CH}=), 6.15(\mathrm{~m}, 1 \mathrm{H}, \mathrm{CH}=), 7.15(\mathrm{~m}$, $1 \mathrm{H}$, ind 5-H), $7.24(\mathrm{~m}, 1 \mathrm{H}$, ind $6-\mathrm{H}), 7.41(\mathrm{~d}, J=8.1 \mathrm{~Hz}, 1 \mathrm{H}$, ind $7-\mathrm{H}), 7.68(\mathrm{dm}, J=8.1 \mathrm{~Hz}, 1 \mathrm{H}$, ind $4-\mathrm{H}) ;{ }^{13} \mathrm{C}$ NMR $\delta 28.5$ $\left(3 \mathrm{CH}_{3}\right), 39.3\left(\mathrm{CH}_{2}\right), 47.9\left(\mathrm{CH}_{2}\right), 55.8\left(\mathrm{CH}_{3}\right), 66.6(\mathrm{CH})$, $74.5\left(\mathrm{CH}_{2}\right), 80.0(\mathrm{C}), 109.5(\mathrm{CH}), 111.6(\mathrm{C}), 115.1\left(\mathrm{CH}_{2}\right)$, $115.9\left(\mathrm{CH}_{2}\right), 119.5(\mathrm{CH}), 120.5(\mathrm{CH}), 122.8(\mathrm{CH}), 128.0$ (C), 134.0(CH), 137.6(C), $137.9(\mathrm{C}), 139.0(\mathrm{CH}), 155.7(\mathrm{CO})$.

4.1.12. 3-[(N-Allyl- $N$-tert-butoxycarbonyl)aminomethyl]2-[1-(tert-butyldimethylsilyloxy)-2-propenyl]-1-(methoxymethyl)indole (13). A solution of alcohol $12(0.21 \mathrm{~g}$, $0.5 \mathrm{mmol})$, TBDMSCl $(0.25 \mathrm{~g}, 1.6 \mathrm{mmol})$, and imidazole $(0.15 \mathrm{~g}, 2.1 \mathrm{mmol})$ in DMF $(3 \mathrm{~mL})$ was heated under $\mathrm{Ar}$ at $55^{\circ} \mathrm{C}$ overnight. The reaction mixture was partitioned between $10 \%$ aqueous $\mathrm{Na}_{2} \mathrm{CO}_{3}$ and $\mathrm{Et}_{2} \mathrm{O}$ and extracted with $\mathrm{Et}_{2} \mathrm{O}$. The organic extracts were dried and concentrated. Flash chromatography (9:1 hexanes-AcOEt) of the residue gave 13: $0.18 \mathrm{~g}$ (64\%); IR (film) $1690 ;{ }^{1} \mathrm{H}$ NMR $\delta 0.19$ (s, 6H), $0.93(\mathrm{~s}, 9 \mathrm{H}), 1.55$ (s, 9H), 3.32 (s, 3H), 3.60 (br s, 2H), 4.77 (br s, 2H), $5.14(\mathrm{~m}, 2 \mathrm{H}), 5.20(\mathrm{dm}, J=9.0 \mathrm{~Hz}, 1 \mathrm{H}), 5.40(\mathrm{~d}$, $J=15.0 \mathrm{~Hz}, 1 \mathrm{H}), 5.54$ and $5.76(2 \mathrm{~d}, J=10.0 \mathrm{~Hz}, 2 \mathrm{H}), 5.77$ $(\mathrm{m}, 2 \mathrm{H}), 6.20(\mathrm{~m}, 1 \mathrm{H}), 7.18(\mathrm{~m}, 1 \mathrm{H}), 7.27(\mathrm{~m}, 1 \mathrm{H}), 7.52(\mathrm{~d}$, $J=7.8 \mathrm{~Hz}, 1 \mathrm{H}), 7.68(\mathrm{~d}, J=7.8 \mathrm{~Hz}, 1 \mathrm{H}) ;{ }^{13} \mathrm{C} \mathrm{NMR} \delta-4.6$ $\left(2 \mathrm{CH}_{3}\right), 18.6(\mathrm{C}), 26.1\left(3 \mathrm{CH}_{3}\right), 28.8\left(3 \mathrm{CH}_{3}\right), 38.7\left(\mathrm{CH}_{2}\right)$, $47.3\left(\mathrm{CH}_{2}\right), 56.0\left(\mathrm{CH}_{3}\right), 68.1(\mathrm{CH}), 75.8\left(\mathrm{CH}_{2}\right), 80.0(\mathrm{C})$, $109.9(\mathrm{C}), 110.8(\mathrm{CH}), 114.6\left(\mathrm{CH}_{2}\right), 116.0\left(\mathrm{CH}_{2}\right), 119.5$ $(\mathrm{CH}), 120.6(\mathrm{CH}), 122.8(\mathrm{CH}), 128.4(\mathrm{C}), 134.1(\mathrm{CH})$, $137.6(\mathrm{CH}), 138.3(\mathrm{C}), 139.9(\mathrm{C}), 155.8$ (CO); HRMS calcd for $\mathrm{C}_{28} \mathrm{H}_{44} \mathrm{~N}_{2} \mathrm{O}_{4} \mathrm{Si} 500.3070$, found 500.3062 .

4.1.13. 3-[( $N$-Allyl- $N$-tert-butoxycarbonyl)aminomethyl]1-(methoxymethyl)-2-propenoylindole (14). Alcohol 12 (39 mg, $0.1 \mathrm{mmol}$ ) and $\mathrm{MnO}_{2}\left(87 \mathrm{mg}, 1.0 \mathrm{mmol}\right.$ ) in $\mathrm{CH}_{2} \mathrm{Cl}_{2}$ $(6 \mathrm{~mL})$ were stirred at $\mathrm{rt}$ for $60 \mathrm{~h}$. The reaction mixture was filtered through Celite and the filtrate was concentrated. The resulting residue was purified by flash chromatography $(8: 2$ hexanes-AcOEt) to give ketone 14: $25 \mathrm{mg}(64 \%) ;{ }^{1} \mathrm{H}$ NMR $\delta 1.50(\mathrm{~s}, 9 \mathrm{H}), 3.19(\mathrm{~s}, 3 \mathrm{H}), 3.55(\mathrm{br} \mathrm{s}, 2 \mathrm{H}), 4.86(\mathrm{~s}, 2 \mathrm{H}), 4.90$ $(\mathrm{dm}, J=15.0 \mathrm{~Hz}, 1 \mathrm{H}), 5.05(\mathrm{dm}, J=9.6 \mathrm{~Hz}, 1 \mathrm{H}), 5.60$ (br s, 1H), 5.64 (s, 2H), 5.99 (dd, $J=10.5,1.5 \mathrm{~Hz}, 1 \mathrm{H}), 6.30$ (dd, $J=17.1,1.5 \mathrm{~Hz}, 1 \mathrm{H}), 6.89(\mathrm{dd}, J=17.1,10.5 \mathrm{~Hz}, 1 \mathrm{H}), 7.22$ (m, 1H), $7.40(\mathrm{~m}, 1 \mathrm{H}), 7.50(\mathrm{~d}, J=7.8 \mathrm{~Hz}, 1 \mathrm{H}), 7.80$ (br d, $J=7.8 \mathrm{~Hz}, 1 \mathrm{H}) ;{ }^{13} \mathrm{C}$ NMR $\delta 28.7\left(3 \mathrm{CH}_{3}\right), 40.0\left(\mathrm{CH}_{2}\right), 47.5$ $\left(\mathrm{CH}_{2}\right), 56.3\left(\mathrm{CH}_{3}\right), 75.4\left(\mathrm{CH}_{2}\right), 80.0(\mathrm{C}), 111.1(\mathrm{CH})$, $116.6\left(\mathrm{CH}_{2}\right), 119.4(\mathrm{C}), 121.8(\mathrm{CH}), 122.2(\mathrm{CH}), 126.2$ $(\mathrm{CH}), 127.3(\mathrm{C}), 131.2\left(\mathrm{CH}_{2}\right), 133.8(\mathrm{CH}), 135.2(\mathrm{C})$, $137.3(\mathrm{CH}), 138.9$ (C), 155.8 (CO), 187.7 (CO); HRMS calcd for $\mathrm{C}_{22} \mathrm{H}_{28} \mathrm{~N}_{2} \mathrm{O}_{4} 384.2049$, found 384.2033.

4.1.14. 2-(tert-Butoxycarbonyl)-6-(tert-butyldimethylsilyloxy)-7-(methoxymethyl)-1,2,3,6-tetrahydroazocino[4,3-b] ]indole (15). Diene $13(0.17 \mathrm{~g}, 0.33 \mathrm{mmol})$ was allowed to react with $\left(\mathrm{PCy}_{3}\right)_{2}(\mathrm{Cl})_{2} \mathrm{Ru}=\mathrm{CHPh}(10 \mathrm{~mol} \%)$ in $\mathrm{CH}_{2} \mathrm{Cl}_{2}(9 \mathrm{~mL})$ as described in Section 4.1.4. After work-up and flash chromatography (95:5 hexanes-AcOEt), compound 15 was obtained: $0.135 \mathrm{~g}(85 \%)$; ${ }^{1} \mathrm{H}$ NMR (400 MHz, gHSQC, 1:1 mixture of rotamers) $\delta 0.07(\mathrm{~s}$, $\left.6 \mathrm{H}, \mathrm{CH}_{3}\right), 0.91\left(\mathrm{~s}, 9 \mathrm{H}, \mathrm{CH}_{3}\right), 1.46$ and $1.49\left(2 \mathrm{~s}, 9 \mathrm{H}, \mathrm{CH}_{3}\right)$, $3.18\left(\mathrm{~s}, 3 \mathrm{H}, \mathrm{OCH}_{3}\right), 3.50,3.75$, and $3.96(3 \mathrm{~m}, 2 \mathrm{H}, 3-\mathrm{H})$, $4.52,4.68,4.87$, and $5.10(4 \mathrm{~d}, J=16.0 \mathrm{~Hz}, 2 \mathrm{H}, 1-\mathrm{H}), 5.45$ and $5.63(2 \mathrm{~m}, 1 \mathrm{H}, 5-\mathrm{H}), 5.63$ and $5.83\left(2 \mathrm{~m}, 2 \mathrm{H}, \mathrm{OCH}_{2}\right)$, 5.91 and $6.03(2 \mathrm{~m}, 1 \mathrm{H}, 4-\mathrm{H}), 6.18(\mathrm{br} \mathrm{s}, 1 \mathrm{H}, 6-\mathrm{H}), 7.16$ (t, $J=8.0 \mathrm{~Hz}, 1 \mathrm{H}, 10-\mathrm{H}), 7.21(\mathrm{t}, J=8.0 \mathrm{~Hz}, 1 \mathrm{H}, 9-\mathrm{H}), 7.45$ $(\mathrm{d}, J=8.0 \mathrm{~Hz}, 1 \mathrm{H}, 8-\mathrm{H}), 7.57(\mathrm{~d}, J=8.0 \mathrm{~Hz}, 1 \mathrm{H}, 11-\mathrm{H})$; ${ }^{13} \mathrm{C}$ NMR (100.6 MHz, gHSQC, $1: 1$ mixture of rotamers) $\delta-4.6\left(2 \mathrm{CH}_{3}\right), 18.4(\mathrm{C}), 26.0\left(3 \mathrm{CH}_{3}\right), 28.7$ and 28.8 $\left(3 \mathrm{CH}_{3}\right), 41.2$ and $41.6(\mathrm{C}-1), 43.9$ and $44.3(\mathrm{C}-3), 55.9$ $\left(\mathrm{OCH}_{3}\right), 66.4$ and $66.5(\mathrm{C}-6), 75.2$ and $75.4\left(\mathrm{CH}_{2} \mathrm{O}\right), 79.8$ and $80.2(\mathrm{C}), 109.5$ and $109.6(\mathrm{C}), 110.2$ and $110.3(\mathrm{C}-8)$, 118.4 and 118.5 (C-11), 120.3 and 120.4 (C-10), 122.3 and 122.4 (C-9), 126.1 and $126.5(\mathrm{C}-5), 127.8$ and $127.9(\mathrm{C})$, 136.1 and $136.3(\mathrm{C}-4), 136.9(\mathrm{C}), 137.3(\mathrm{C}), 155.3$ and 155.4 (CO); HRMS calcd for $\mathrm{C}_{26} \mathrm{H}_{40} \mathrm{~N}_{2} \mathrm{O}_{4} \mathrm{Si}$ 472.2757, found 472.2750 .

4.1.15. 2-(tert-Butoxycarbonyl)-7-(methoxymethyl)6-oxo-1,2,3,6-tetrahydroazocino $[4,3-b]$ indole (16). (Im)$\left(\mathrm{PCy}_{3}\right)(\mathrm{Cl})_{2} \mathrm{Ru}=\mathrm{CHPh}$ (second generation Grubbs catalyst, $10 \mathrm{~mol} \%$ ) was added under Ar to a solution of ketone 14 (25 mg, $0.065 \mathrm{mmol})$ in $\mathrm{CH}_{2} \mathrm{Cl}_{2}(2 \mathrm{~mL})$ and the resulting mixture was stirred at rt overnight. The reaction mixture was filtered, the filtrate was concentrated, and the resulting residue was purified by flash chromatography (8:2 hexanesAcOEt) to give 16: $20 \mathrm{mg}(86 \%) ;{ }^{1} \mathrm{H}$ NMR (400 MHz) $\delta 1.48$ (br s, 9H), 3.27 (s, 3H), 3.91 (br s, 2H), 4.83 (s, 2H), 5.99 (s, 2H), $6.44(\mathrm{~m}, 1 \mathrm{H}), 6.63(\mathrm{~d}, J=11.7 \mathrm{~Hz}, 1 \mathrm{H})$, $7.26(\mathrm{~m}, 1 \mathrm{H}), 7.43(\mathrm{~m}, 1 \mathrm{H}), 7.55(\mathrm{~d}, J=8.4 \mathrm{~Hz}, 1 \mathrm{H}), 7.90$ $(\mathrm{m}, 1 \mathrm{H}) ;{ }^{13} \mathrm{C}$ NMR $(100.6 \mathrm{MHz}) \delta 28.8\left(3 \mathrm{CH}_{3}\right), 37.2$ $\left(\mathrm{CH}_{2}\right), 39.6\left(\mathrm{CH}_{2}\right), 56.3\left(\mathrm{CH}_{3}\right), 75.4\left(\mathrm{CH}_{2}\right), 81.1(\mathrm{C})$, $111.6(\mathrm{CH}), 121.4(\mathrm{CH}, \mathrm{C}), 122.0(\mathrm{CH}), 126.5(\mathrm{C}), 127.6$ (CH), $133.7(\mathrm{C}), 135.7(\mathrm{CH}), 138.4(\mathrm{C}), 139.9(\mathrm{CH}), 154.6$ (CO), 184.2 (CO). Anal. Calcd for $\mathrm{C}_{20} \mathrm{H}_{24} \mathrm{~N}_{2} \mathrm{O}_{4} \cdot 1 / 2 \mathrm{H}_{2} \mathrm{O}$ : C, $65.74 \%$; H, 6.90\%; N, 7.67\%. Found: C, 65.85\%; H, $6.66 \% ; \mathrm{N}, 7.65 \%$.

4.1.16. 2-(tert-Butoxycarbonyl)-6-(tert-butyldimethylsilyloxy)-7-(methoxymethyl)-1,2,3,4,5,6-hexahydroazocino[4,3-b]indole (17). Compound 15 (63 mg, $0.13 \mathrm{mmol})$ dissolved in $\mathrm{MeOH}(6 \mathrm{~mL})$ was hydrogenated over $\mathrm{Pd} / \mathrm{C}$ $(5 \%, 3.5 \mathrm{mg})$ for $12 \mathrm{~h}$. The catalyst was filtered, the filtrate was concentrated, and the resulting residue was purified by flash chromatography ( $8: 2$ hexanes-AcOEt) to give azocinoindole 17: $51 \mathrm{mg}(80 \%) ;{ }^{1} \mathrm{H}$ NMR $(400 \mathrm{MHz}) \delta 0.08$ (s, $3 \mathrm{H}), 0.93(\mathrm{~s}, 9 \mathrm{H}), 1.46$ and $1.55(2 \mathrm{~s}, 9 \mathrm{H}), 1.8(\mathrm{~m}, 2 \mathrm{H}), 2.15$ $(\mathrm{m}, 1 \mathrm{H}), 2.90(\mathrm{~m}, 1 \mathrm{H}), 3.23(\mathrm{~s}, 3 \mathrm{H}), 3.50$ and $3.85(2 \mathrm{~m}, 2 \mathrm{H})$, $4.85(\mathrm{~m}, 2 \mathrm{H}), 5.51(\mathrm{~m}, 1 \mathrm{H}), 5.56$ and $5.68(2 \mathrm{~d}, J=14.4 \mathrm{~Hz}$, 2H), 7.10-7.26 (m, 2H), $7.42(\mathrm{~d}, J=8.0 \mathrm{~Hz}, 1 \mathrm{H}), 7.75(\mathrm{~m}$, $1 \mathrm{H}) ;{ }^{13} \mathrm{C}$ NMR $\left(1: 1\right.$ mixture of rotamers) $\delta-4.64\left(2 \mathrm{CH}_{3}\right)$, $18.5(\mathrm{C}), 24.0$ and $24.1\left(\mathrm{CH}_{2}\right), 26.2\left(3 \mathrm{CH}_{3}\right), 29.0\left(3 \mathrm{CH}_{3}\right)$, 36.9 and $37.3\left(\mathrm{CH}_{2}\right), 39.4$ and $39.7\left(\mathrm{CH}_{2}\right), 43.9$ and 44.2 $\left(\mathrm{CH}_{2}\right), 56.0\left(\mathrm{CH}_{3}\right), 67.4(\mathrm{CH}), 74.6\left(\mathrm{CH}_{2}\right), 79.9(\mathrm{C}), 109.5$ and $109.7(\mathrm{CH}), 110.4$ and $110.5(\mathrm{C}), 119.1$ and 119.5 $(\mathrm{CH}), 120.3$ and $120.5(\mathrm{CH}), 122.4(\mathrm{CH}), 127.99$ and 128.5 (C), 137.5 (C), 139.5 (C), 155.4 (CO); HRMS calcd for $\mathrm{C}_{26} \mathrm{H}_{42} \mathrm{~N}_{2} \mathrm{O}_{4} \mathrm{Si} 474.7083$, found 474.7078 .

4.1.17. 2-(tert-Butoxycarbonyl)-6-oxo-7-(methoxymethyl)-1,2,3,4,5,6-hexahydroazocino[4,3-b]indole (18). Operating as above, from $16(0.28 \mathrm{~g}, 0.78 \mathrm{mmol})$ azocinoindole $\mathbf{1 8}$ was obtained after flash chromatography (6:4 hexanes-AcOEt): $0.23 \mathrm{~g}(82 \%) ;{ }^{1} \mathrm{H}$ NMR (1:1 mixture of rotamers) 1.19 and $1.45(2 \mathrm{~s}, 9 \mathrm{H}), 2.10(\mathrm{br}, 2 \mathrm{H}), 2.95(\mathrm{br}$, $2 \mathrm{H}), 3.21(\mathrm{~s}, 3 \mathrm{H}), 3.55$ and $3.65(2 \mathrm{~m}, 2 \mathrm{H}), 4.80$ and 4.90 (2br s, 2H), 5.73 (br, 2H), 7.20 (t, $J=8.0 \mathrm{~Hz}, 1 \mathrm{H}), 7.38$ (t, $J=8.0 \mathrm{~Hz}, 1 \mathrm{H}), 7.50$ (br d, $J=8.0 \mathrm{~Hz}, 1 \mathrm{H}), 7.70(\mathrm{~m}, 1 \mathrm{H})$; ${ }^{13} \mathrm{C}$ NMR (1:1 mixture of rotamers) 25.3 and $25.6\left(\mathrm{CH}_{2}\right)$, 
28.2 and $28.3\left(3 \mathrm{CH}_{3}\right), 41.7$ and $41.9\left(\mathrm{CH}_{2}\right), 43.4$ and 44.1 $\left(\mathrm{CH}_{2}\right), 46.1$ and $47.9\left(\mathrm{CH}_{2}\right), 55.8\left(\mathrm{CH}_{3}\right), 74.7\left(\mathrm{CH}_{2}\right), 80.0$ (C), 110.7 and $110.9(\mathrm{CH}), 120.2$ and $120.5(\mathrm{CH}), 121.3$ and $121.4(\mathrm{CH}), 122.3(\mathrm{C}), 125.8$ and $126.0(\mathrm{CH}, \mathrm{C})$, $132.5(\mathrm{C}), 137.8(\mathrm{C}), 155.4(\mathrm{CO}), 197.6$ and $198.2(\mathrm{CO})$; HRMS calcd for $\mathrm{C}_{20} \mathrm{H}_{26} \mathrm{~N}_{2} \mathrm{O}_{4} 358.1892$, found 358.1887.

\section{Acknowledgements}

Financial support from the 'Ministerio de Ciencia y Tecnología' (MCYT-FEDER, Spain) through project BQU2003-04967-C-02-02 is gratefully acknowledged.

\section{References and notes}

1. General reviews: (a) Handbook of Metathesis; Grubbs, R. H., Ed.; Wiley-VCH: Weinheim, 2003; Vol. 2; (b) Nicolaou, K. C.; Bulger, P. G.; Sarlah, D. Angew. Chem., Int. Ed. 2005, $44,4490-4527$.

2. For recent reviews on the application of RCM to the synthesis of heterocycles, see: (a) Walters, M. A. Progress in Heterocyclic Chemistry; Gribble, G. W., Joule, J. A., Eds.; Pergamon: Amsterdam, 2003; Vol. 15, pp 1-36; (b) Nakamura, I.; Yamamoto, Y. Chem. Rev. 2004, 104, 2127-2198; (c) Deiters, A.; Martin, S. F. Chem. Rev. 2004, 104, 2199-2238.

3. (a) Maier, M. E. Angew. Chem., Int. Ed. 2000, 39, 2073-2077; (b) Yet, L. Chem. Rev. 2000, 100, 2963-3007; (c) Michaut, A.; Rodriguez, J. Angew. Chem., Int. Ed. 2006, 45, 5740-5750.

4. For previous examples of RCM of dienes on indole nuclei, see: (a) Birman, V. B.; Rawal, V. H. J. Org. Chem. 1998, 63, 91469147; (b) González-Pérez, P.; Pérez-Serrano, L.; Casarrubios, L.; Domínguez, G.; Pérez-Castells, J. Tetrahedron Lett. 2002, 43, 4765-4767; (c) Chacun-Lefèvre, L.; Bénéteau, V.; Joseph, B.; Mérour, J.-Y. Tetrahedron 2002, 58, 1018110188; (d) Kalinin, A.; Chauder, B. A.; Rahkit, S.; Snieckus, V. Org. Lett. 2003, 5, 3519-3521; (e) Bremmer, J. B.; Coates, J. A.; Keller, P. A.; Pyne, S. G.; Witchard, H. M. Tetrahedron 2003, 59, 8741-8755; (f) Yang, X.; Althammer, A.; Knochel, P. Org. Lett. 2004, 6, 1665-1667; (g) Pelly, S. C.; Parkinson, C. J.; van Otterlo, W. A. L.; de Koning, C. B. J. Org. Chem. 2005, 70, 10474-10481.
5. For specific examples of enyne RCM, see: (a) Schramm, M.-P.; Reddy, D. S.; Kozmin, S. A. Angew. Chem., Int. Ed. 2001, 49, 4274-4277; (b) Pérez-Serrano, L.; Casarrubios, L.; Domínguez, G.; Freire, G.; Pérez-Castells, J. Tetrahedron 2002, 58, 5407-5415; (c) González-Gómez, A.; Domínguez, G.; Pérez-Castells, J. Tetrahedron Lett. 2005, 46, 7267-7270.

6. (a) Sundberg, R. J. Indoles; Academic: New York, NY, 1996; (b) Joule, J. A. Science of Synthesis (Houben-Weyl, Methods of Molecular Transformations); Georg Thieme: Stuttgart, 2000; Vol. 10, pp 361-652; (c) Somei, M.; Yamada, F. Nat. Prod. Rep. 2005, 22, 73-103 and 761-793.

7. Joule, J. A. Indoles, The Monoterpenoid Indole Alkaloids; Saxton, J. E., Ed.; Wiley: New York, NY, 1983; Vol. 25, pp 265-292.

8. For pioneering synthetic approaches to apparicine, see: Joule, J. A.; Allen, M. S.; Bishop, D. I.; Harris, M.; Hignett, G. J.; Scopes, D. I. C.; Wilson, N. D. V. Indole and Biogenetically Related Alkaloids; Phillipson, J. D., Zenk, M. H., Eds.; Academic: London, 1980; pp 229-247.

9. Rahman, A. U.; Muzaffar, A. Heterocycles 1985, 23, 29752988.

10. For a preliminary communication of this part of the work, see: Bennasar, M.-L.; Zulaica, E.; Tummers, S. Tetrahedron Lett. 2004, 45, 6283-6285.

11. Kondo, Y.; Takazawa, N.; Yoshida, A.; Sakamoto, T. J. Chem. Soc., Perkin Trans. 1 1995, 1207-1208.

12. Saulnier, M. G.; Gribble, G. W. J. Org. Chem. 1982, 47, 757-761.

13. Gribble, G. W.; Keavy, D. J.; Davis, D. A.; Saulnier, M. G.; Pelcman, B.; Barden, T. C.; Sibi, M. P.; Olson, E. R.; BelBruno, J. J. J. Org. Chem. 1992, 57, 5878-5891.

14. Comins, D. L.; Killpack, M. O. J. Org. Chem. 1987, 52, 104-109.

15. For a previous approach to azocino[4,3- $b]$ indoles, see: Street, J. D.; Harris, M.; Bishop, D. I.; Heatley, F.; Beddoes, R. L.; Mills, O. S.; Joule, J. A. J. Chem. Soc., Perkin Trans. 1 1987, 1599-1606. See also Ref. 8.

16. For metal-mediated syntheses of related azocino[5,4- $b]$ indoles, see: (a) Baran, P. S.; Corey, E. J. J. Am. Chem. Soc. 2002, 124, 7904-7905; (b) Baran, P. S.; Guerrero, C. A.; Corey, E. J. J. Am. Chem. Soc. 2003, 125, 5628-5629; (c) Watanabe, T.; Arai, S.; Nishida, A. Synlett 2004, 907-909; (d) Ferrer, C.; Echavarren, A. M. Angew. Chem., Int. Ed. 2006, 45, 1105-1109. 